\title{
3 Research Square \\ CircRNA Expression in Chicken Granulosa Cells Illuminated with Red Light
}

Ying Wang

Yangzhou University

Zhenyu Guo

Yangzhou University

Chen Zi

Linyi People's Hospital

Pengfei Wu

Yangzhou University

Xiaoyang Lv

Yangzhou University

Lan Chen

Yangzhou University

Fuxiang Chen

Yangzhou University

Genxi Zhang

Yangzhou University

Jinyu Wang ( $\sim$ jywang@yzu.edu.cn )

Yangzhou University

\section{Research article}

Keywords: circRNA, chicken, monochromatic light, granulosa cell, small yellow follicle

Posted Date: August 20th, 2020

DOI: https://doi.org/10.21203/rs.3.rs-52557/v1

License: (c) (i) This work is licensed under a Creative Commons Attribution 4.0 International License.

Read Full License

Version of Record: A version of this preprint was published at Poultry Science on January 1st, 2022. See the published version at https://doi.org/10.1016/j.psj.2022.101734. 


\section{Abstract}

Background Our previous work revealed that red light (RL) can improve egg production by Jinghai Yellow hens. Circular RNAs (circRNAs) are a novel non-coding RNAs, but the molecular mechanism underpinning their functions during follicular development of hens under monochromatic light remains unknown.

Results We compared expression profiles of granulosa cells (GCs) of small yellow follicles (SYFs) from hens under $R L$ and white light (WL). A total of 2,468 circRNAs were identified, of which 22 were differentially expressed (DE) between RL and WL groups. Host genes of DE circRNAs were enriched in ovarian steroidogenesis, and MAPK signalling and PI3K-Akt signalling pathways. Furthermore, DE circRNA_0320 and circRNA_0185 interact with miR-143-3p targeting FSHR, which is essential for GC differentiation and follicle development.

Conclusions These findings will facilitate further analysis of the molecular mechanism of circRNAs in GCs of SYFs, and expand our understanding of follicle development, which could lead to increased egg production.

\section{Background}

The rate of follicle development is closely related to egg production by hens [1], and the reproductive strategy for avian species that produce clutches of eggs is dependent upon the maintenance of a small cohort of viable, undifferentiated follicles [2]. In hens, one small yellow follicle (SYF) of 6-8 $\mathrm{mm}$ in diameter is selected daily from a pool of follicles of similar size to become a preovulatory follicle [3-4]. Furthermore, the proliferation and differentiation of granulosa cells (GCs) are closely related to follicular development [5], and Orisaka et al. (2009) demonstrated that follicular growth is tightly regulated by intraovarian oocyte-granulosa-theca cell interactions [6]. GCs help to supply nutrients during oocyte growth through gap junction associations [7-8], and Shen et al. (1993) reported that yolk particles passing between GCs are important for the growth of chicken oocytes [9-10].

The SYF contains significantly more follicle stimulating hormone receptor (FSHR) than the other follicles [11], which is one of the earliest markers for the most recently 'selected' follicle in the granulosa layer of the hen ovary [4]. However, the granulosa layer within prehierarchical follicles remains undifferentiated and steroidogenically inactive [12]. Tilly et al. (1991) demonstrated that the steroidogenic competency of GCs occurs during the transition of follicles from 6-8 $\mathrm{mm}$ to $9-12 \mathrm{~mm}$ [13]. Differentiated GCs derived from preovulatory follicles are capable of producing progesterone, particularly in response to $\mathrm{LH}$, which eventually results in an LH surge that initiates ovulation [14-15]. Therefore, follicle selection is associated with an increase in P450scc, together with progesterone secretion in the GCs of SYFs [5], hence the final differentiation of the GC layer commences at the time of follicle selection [12].

Circular RNAs (circRNAs) are novel non-coding RNAs that are ubiquitously expressed in eukaryotic cells during post-transcriptional processes [16]. CircRNAs are unusually stable RNAs produced by circularisation of exons through a poorly characterised mechanism [17]. Memczak et al. (2013) detected 
thousands of highly expressed, stable circRNAs that often display tissue- and/or developmental-stagespecific expression [18]. Endogenous circRNA molecules can act as efficient miRNA sponges, adding to a growing repertoire of known regulatory functions in gene expression [19-20], and they also modulate premRNA alternative splicing, and possess protein-coding capacity [21]. Wu et al. (2020) found that host genes of circRNAs in GCs of duck preovulatory follicles are enriched in vital pathways related to oocyte development and cell proliferation [22]. Shen et al. (2019) identified the key gene RalGPS2 which is produced in three isoforms of circRNAs in GCs of chicken follicles [23]. Artificial illumination is widely used in poultry, and our previous studies revealed that egg production of hens aged 300 days under RL was 90.61 eggs, significantly higher than that of the WL group (87.44 eggs). Thus, RL significantly improves egg production by Jinghai Yellow hens, but the molecular mechanism underpinning the functions of circRNAs during follicular development of hens under RL remains unknown.

In the present study, RNA sequencing (RNA-seq) was performed to identify circRNAs in GCs of SYFs of Jinghai Yellow chickens under monochromatic light. The results will prove useful for exploring the molecular mechanism of GC development in hens under monochromatic light.

\section{Results}

\section{Identification of circRNAs in GCs of SYFs in hens}

Six circRNA libraries were built from RL $(n=3)$ and WL $(n=3)$ groups, an Illumina Hiseq 4000 instrument was applied to sequence these samples, and the obtained reads were mapped to the chicken reference genome (Gallus-gallus-5.0/galGal5). After removing low-quality sequences and adapters, $55.35 \mathrm{~Gb}$ of clean reads was obtained with an average GC content of $48.2 \%$. CIRI and circBase were used to identify circRNAs [24-25], and 2,468 circRNAs were identified in regenerating GCs of SYFs in RL and WL groups. Nearly $85 \%$ of circRNAs had a predicted splice length of $<2,000$ bp, $46.64 \%$ of circRNAs had a length $>500$ bp, $26.26 \%$ were $500-1000 \mathrm{bp}$, and the average length was 2,564 bp (Fig. 1A). The identified circRNAs in GCs were distributed across almost all chromosomes, with the fewest on chromosome (chr) 32 and the most on chr1 (Fig. 1B). According to their genomic loci, circRNAs were grouped into exon, intron and intergenic regions. Most $(2,180)$ circRNAs originated from sense genic_exonic regions (Fig. 1C).

\section{Analysis of DE circRNAs}

Expression levels of circRNAs of GC libraries of SYFs were compared between RL and WL groups, and 22 DE circRNAs were identified $(p<0.05)$. Of these, 15 were upregulated and 7 were downregulated (Fig. 2A, Additional File 1). Furthermore, spliced reads per million (RPM) was used to measure expression levels of circRNAs [26], and volcano plots of DE circRNAs were plotted (Fig. 2B).

\section{GO and KEGG analyses of host genes related to DE circRNAs}

The biological functions of host genes related to DE circRNAs were further analysed. GO enrichment analysis was performed on DE circRNAs involved in the positive regulation of cell migration, the ERK1and 
ERK2 cascade, cell-cell signalling, and inositol 1,4,5-trisphosphate binding (Fig. 3A, Additional file 2). KEGG pathway enrichment analysis of DE circRNAs identified ovarian steroidogenesis, as well as MAPK signalling, prolactin signalling, PI3K-Akt signalling and thyroid hormone signalling pathways (Fig. 3B, Additional file 3).

\section{Verification of DE circRNAs and host genes by RT-qPCR}

To validate the expression levels of DE circRNAs and host genes to support further analysis of the molecular mechanism of monochromatic light on follicular development in hens, five circRNAs (circRNA_1946, circRNA_0320, circRNA_0185, circRNA_0007, and circRNA_1481) and five genes (FSHR, EGF-like fibronectin type III and laminin G domain [EGFLAM], mitogen-activated protein kinase kinase kinase 5 [MAP3K5], phosphoinositide-3-kinase regulatory subunit 1 [PIK3R1], photopigment melanopsinlike [OPN4-1]) were analysed by RT-qPCR, and the results were compared with the high-throughput RNAseq results (Fig. 4). The WL group served as a control group, and the expression pattern revealed that the two methods gave consistent results.

\section{Coexpression networks of predicted target miRNAs and genes}

It is well established that one of the main functions of circRNAs is acting as miRNA sponges [19], and circRNA-miRNA interactions can predict the characteristics of circRNAs [20]. Herein, 24 miRNAs and 193 mRNAs were predicted by miRBase [52], and the results are shown in Additional files 4 and 5. Cytoscape software was applied to construct circRNA-miRNA-mRNA coexpression networks [53], and circRNA_1805 and circRNA_0007 were found to share the common targets miR-425-5p, circRNA_0320, and circRNA_1805, with the same 11 miRNAs, including miR-143-3p, miR-142-3p, miR-107-3p, miR-27b-3p, and miR-460a-5p. The molecular interactions between these three circRNAs and their miRNAs and target genes are illustrated in Figure 5. Target genes including FSHR, EGFLAM, MAP3K5 and PIK3R1 are related to chicken reproductive performance.

\section{Discussion}

Chicken ovaries are considered an excellent model of follicular selection because they the size of follicles can be easily observed [3]. Many of the processes of follicle growth and differentiation are initiated specifically within the GC layer [2]. In order to increase egg production by hens, we investigated the effect of monochromatic light on reproductive performance. Comparing WL and RL groups revealed a significant increase in egg production by Jinghai Yellow chickens, and this could have economic benefits. Exploring the follicular development of prehierachical follicles (SYFs) of hens in response to monochromatic light could assist the modern poultry industry. CircRNAs are ubiquitous in the transcriptome of humans and mice, and circRNA expression is a feature of all eukaryotes [27-29]. Most circRNA sponges are formed through backsplicing, and are enriched in miRNA-binding sites [30-31]. RL increases egg production by Jinghai Yellow chickens, therefore we analysed DE circRNAs, miRNAs, and mRNAs of GCs of SYFs of Jinghai Yellow chickens under RL and WL exposure to identify the key circRNAs involved in follicular development. 
The identified DE circRNAs were widely distributed across most chromosomes, with the most on chr1 and the fewest on chr 32, in accordance with previous studies [23]. A total of 2,468 circRNAs were identified in GCs of SYFs, which is more than the number in liver of ALV-J-resistant chickens $(1,800)$ [32] but less than the number in spleen of ALV-J-infected chickens $(4,254)$ [33], possibly due to tissue-specific factors. In total, 11,642 circRNAs of three types of GCs (SYF, F1, and F6) of chicken [23] and 4,204 circRNAs of two types of GCs (SYFs and large white follicles (LWFs)) were identified for duck follicles [22], both considerably more than identified herein, which indicates that the number of circRNAs may be closely correlated with the number of types of follicles. We found that the most common lengths for circRNAs were 200-300 nt and >2,000 nt, and Shen et al. (2019) similarly found that the majority of circRNAs were 200-300 nt, but lengths $>1400 \mathrm{nt}$ were not mentioned [23]. In the present study, we identified $22 \mathrm{DE}$ circRNA in GCs of SYFs between RL and WL groups (Fig. 2). Wu et al. (2020) previously identified 14 DE circRNAs of GCs between LWFs and SYFs [22], while Shen et al. (2019) identified 219 DE circRNAs of GCs between SYFs and F6 [23], indicating that the number of DE circRNAs is closely related to the contract group. Moreover, host genes of DE circRNAs were enriched in ovarian steroidogenesis, as well as MAPK signalling, prolactin signalling and PI3K-Akt signalling pathways. Previous studies concluded that ovarian steroidogenesis is important for GC differentiation and follicle development [12, 34-35]. MAPK signalling prevents premature differentiation of the granulosa layer within prehierarchical follicles [36], which is involved in the regulation of follicle growth. The PI3K pathway is known to be essential for the regulation of cell proliferation, survival, and migration, is also likely to play a role in regulation of the activation of primordial follicles in the mouse ovary [37]. It can be concluded that DE circRNAs play an important role in GC development and follicluar maturation.

Previous studies have shown that chr5:13061358|13064218 binds to miR-27b-3p and participates in follicular development of chicks [23], and aplacirc_013267 directly binds to and inhibits miR-1-13 in duck ovarian follicles [22]. In the present study, we found that circRNA_0320 and circRNA_0185 were differentially expressed between $\mathrm{RL}$ and $\mathrm{WL}$ groups, and a number of miRNAs were predicted to interact with these circRNAs, including miR-27b-3p, miR-143-3p and miR-142-3p (Fig. 5). Furthermore, the circRNAmiRNA-mRNA network also showed that miR-143p-3p targets FSHR, in accordance with Zhang et al. (2018) and Du et al. (2016) who concluded that miR-143 affects GC apoptosis by binding to FSHR [3839]. One of the earliest markers for differentiating GCs is elevated expression of FSHR [36]; FSHR mRNA levels in the granulosa layer were highest within the cohort of 6-8 $\mathrm{mm}$ follicles (SYFs) during hen follicle development [40], in accordance with our study showing that expression of FSHR was higher in GCs of SYFs of Jinghai Yellow chicken. Genes including EGFLAM, MAP3K5, PIK3R1 and OPN4-1 were identified as targets of DE circRNAs, and these play an important role in follicular development. MAP3K5 is a reliable marker for dominant follicles in cattle, and at day 2.5 post-ovulation there are clear differences in expression of MAP3K5 between the dominant follicle and the next largest follicle a day later [41-42]. Panda et al. (2002) concluded that Opn4 is required for light-induced circadian phase shifting, and OPNs play a pivotal role in non-image-forming responses to light, including physiological adaptations to ambient light [43-44]. PIK3R1 plays an important role in regulating ovarian activities in yak seasonal reproduction [45]. EGFLAM is involved in cellular growth, differentiation and proliferation, including 
angiogenesis and membrane fusion [46]. Competing endogenous RNAs (ceRNAs) analysis revealed that circRNAs can compete with miRNAs to alter the expression of key genes involved in GC differentiation and follicle development. Further characterisation of the functions of circRNAs and associated molecules related to follicular development are required to confirm the roles of ceRNAs in GC differentiation, and interference studies could prove useful for this purpose.

\section{Conclusions}

In conclusion, 2,468 circRNAs were identified in the GC of SYFs of Jinghai Yellow chickens under RL and WL exposure. Furthermore, DE circRNA_0320 and circRNA_0185 were found to interact with miR-143-3p that targets FSHR, and essential protein for GC differentiation. These findings will facilitate further analysis of the molecular mechanism of circRNAs in GCs, and provide insight into ceRNA interactions in follicular development, which could help to improve egg production by Jinghai Yellow chickens.

\section{Methods}

\section{Ethics approval}

This study was reviewed and approved by the Institutional Animal Care and Use Committee of the Department of Animal Science and Technology, Yangzhou University, China, and all chicken procedures were performed according to the Standards for the Administration of Experimental Practices (Jiangsu, China, 2008).

\section{Chicken rearing and sample preparation}

Jinghai Yellow Chickens were raised in Jiangsu Jinghai Poultry Industry Group Co., Ltd. (Nantong, Jiangsu, China). After transfer to the laying house, hens were caged individually and provided with water ad libitum and restricted food. A total of 300 hens were divided into two groups and exposed to a red LED $(\mathrm{RL}, 660 \mathrm{~nm})$ or a white LED (WL, 400-760 nm) for $16 \mathrm{~h}$ each day, with five subgroups in each group. The light intensity was 15.0 lux as measured with a TES-1336A light meter (TES Electrical Electronic Crop., Taipei, China). Based on the pedigree record, six half-sibhens with an average body weight at 300 days of age were selected. The hens were properly anesthetized with isoflurane and decapitated, SYFs were collecte and washed carefully in cold phosphate-buffered saline (PBS; Gibco, USA) and collected. GCs were collected as described previously [47], flash-frozen in liquid nitrogen, and stored at $-80^{\circ} \mathrm{C}$.

\section{RNA-seq preparation and sequencing}

TRIzol reagent (Invitrogen, USA) was used to extract total RNA of GCs of SYFs, $1 \%$ agarose gel electrophoresis was performed to assess the quality of total RNA, and a spectrophotometer at wavelengths of 260 and $280 \mathrm{~nm}$ were applied to measure the concentration, purity and mass. Samples with an RNA integrity number $>9.0$ and an OD 260/280 value of $~ 2.0$ were used. A total of $5 \mu \mathrm{g}$ RNA from each sample was used to construct the circRNA library. Ribosomal RNA was removed, the library was 
built, and high-throughput RNA sequencing was performed by Shanghai OE Biotech Co., Ltd. (Shanghai, China).

\section{Identification of differentially expressed circRNAs}

Bioinformatic methods were performed for quality control, and Q20, Q30 and GC content were calculated. Clean reads were aligned to the reference genome by Bowtie2 and HISAT2 software [48]. CircRNAs were identified by find_circ [49] and CIRI2 [50], and expression levels of circRNAs were measured by mapped backsplicing junction reads per million mapped reads. The DESeq package, fold changes of the same circRNA in two groups $\geq 2$, and $p$-value $\leq 0.05$ were used to distinguish DE circRNAs.

\section{Bioinformatics analysis}

The potential functions of host genes of DE circRNAs and predicted target genes were analysed. The clusterProfiler package within R software was used for GO and KEGG pathway annotation [51]. Interactions of miRNAs and circRNAs were analysed by miRanda with a score of ${ }^{3} 140$ as a cutoff. Targetscan and miRDB were used to predict target genes of miRNAs [52], and Cytoscape software was applied to describe the miRNA-circRNA network [53].

\section{RT-qPCR validation}

Total RNA was extracted from GCs of SYFs of hens using a mirVana RNA Isolation Kit (ABI, USA) according to the manufacturer's specifications. Quantification was performed with a two-step reaction, and each reverse transcription reaction consisted of $0.5 \mu \mathrm{g}$ RNA, $2 \mu \mathrm{L}$ of $5 \times$ TransScript All-in-one SuperMix for qPCR, and $0.5 \mu \mathrm{L}$ of gDNA Remover in a total volume of $10 \mu \mathrm{L}$. Reactions were performed using a GeneAmp PCR System 9700 (Applied Biosystems, USA) for $15 \mathrm{~min}$ at $42^{\circ} \mathrm{C}$ and $5 \mathrm{~s}$ at $85^{\circ} \mathrm{C}$. The $10 \mu \mathrm{L}$ reverse transcription reaction mix was then diluted 10 -fold in nuclease-free water and held at $-20^{\circ} \mathrm{C}$. PCR products for CDNA and genomic DNA were analysed by agarose gel electrophoresis and backsplicing sites of circRNAs were confirmed by Sanger sequencing at Sango Biotech Co. Ltd. (Shanghai, China). Primer sequences were designed according to previous studies [22-23], and primers for circRNAs and mRNAs are listed in Additional file 6. RT-qPCR was performed using a LightCycler 480 II real-time PCR Instrument (Roche, Switzerland) using a $10 \mu \mathrm{L}$ reaction mixture containing $1 \mu \mathrm{L}$ of cDNA, 5 $\mu \mathrm{L}$ of $2 \times$ PerfectStart Green qPCR SuperMix, $0.2 \mu \mathrm{L}$ of forward primer, $0.2 \mu \mathrm{L}$ of reverse primer, and $3.6 \mu \mathrm{L}$ of nuclease-free water. Reactions were incubated in a 384-well optical plate (Roche) at $94^{\circ} \mathrm{C}$ for $30 \mathrm{~s}$, followed by 45 cycles at $94^{\circ} \mathrm{C}$ for $5 \mathrm{~s}$ and $60^{\circ} \mathrm{C}$ for $30 \mathrm{~s}$. Each sample was run in triplicate for analysis. Using ACTB as a reference, relative expression levels of genes and IncRNAs were quantified using the $2^{-\triangle \Delta C T}$ method [54].

\section{Abbreviations}

RL

red light 
WL

white light

GC

granulosa cells

SYF

small yellow follicle

DE

differentially expressed

Chr

chromosome

FSHR

follicle stimulating hormone receptor

EGFLAM

EGF-like fibronectin type III and laminin G domain

MAP3K5

mitogen-activated protein kinase kinase kinase 5

PIK3R1

phosphoinositide-3-kinase regulatory subunit 1

OPN4-1

photopigment melanopsin-like

\section{Declarations}

\section{Ethics approval and consent to participate}

This study was reviewed and approved by the Institutional Animal Care and Use Committee of the Department of Animal Science and Technology, Yangzhou University, China, and all chicken procedures were performed according to the Standards for the Administration of Experimental Practices (Jiangsu, China, 2008).

Consent for publication

Not applicable.

\section{Availability of data and material}

RNA sequence data is uploading.

\section{Competing interests}

The author(s) declare no competing financial interests. 


\section{Funding}

This study was funded by the Priority Academic Program Development of Jiangsu Higher Education Institutions, China Agricultural Research Systems (grant No. CARS-41). The funding body did not have any role in the study design, data collection, analysis and interpretation.

\section{Authors' contributions}

Conceptualisation, Y.W., J.W., and G.Z.; methodology, Y.W., Z.G., P.W., X.L, and L.C.; validation, Y.W.; writing -original draft preparation, Y.W.; writing-review and editing, J.W.; supervision, J.W., and G.Z.; funding acquisition, J.W. All authors have read and agreed to the published version of the manuscript.

\section{Acknowledgments}

Not applicable.

\section{References}

1. Johnson PA, Stephens CS, Giles JR. The domestic chicken: causes and consequences of an egg a day. Poult Sci. 2015;94:816-20.

2. Johnson AL. Ovarian follicle selection and granulosa cell differentiation. Poult Sci. 2015;94:781-5.

3. Hernandez AG, Bahr JM. Role of FSH and epidermal growth factor (EGF) in the initiation of steroidogenesis in granulosa cells associated with follicular selection in chicken ovaries. Reproduction. 2003;125:683-91.

4. Woods DC, Johnson AL. Regulation of follicle-stimulating hormone-receptor messenger RNA in hen granulosa cells relative to follicle selection. Biol Reprod. 2005;72:643-50.

5. Jin Y, Zhang C, Lin X, Zeng W. Prostaglandin involvement in follicle-stimulating hormone-induced proliferation of granulosa cells from chicken prehierarchical follicles. Prostaglandins Other Lipid Mediat. 2006;81:45-54.

6. Orisaka M, Tajima K, Tsang BK, Kotsuji F. Oocyte-granulosa-theca cell interactions during preantral follicular development. J Ovarian Res. 2009;2:9.

7. Larsen WJ, Tung HN, Murray SA, Swenson CA. Evidence for the participation of actin microfilaments and bristle coats in the internalization of gap junction membrane. J Cell Biol. 1979;83:576-87.

8. Buccione R, Schroeder AC, Eppig JJ. Interactions between somatic cells and germ cells throughout mammalian oogenesis. Biol Reprod. 1990;43:543-7.

9. Shen X, Steyrer E, Retzek H, Sanders EJ, Schneider WJ. Chicken oocyte growth: receptor-mediated yolk deposition. Cell Tissue Res. 1993;272:459-71. 
10. Schneider WJ. Receptor-mediated mechanisms in ovarian follicle and oocyte development. Gen Comp Endocrinol. 2009;163:18-23.

11. You S, Bridgham JT, Foster DN, Johnson AL. Characterization of a chicken follicle-stimulating hormone receptor (cFSH-R) cDNA, and expression of cFSH-R mRNA in the ovary. Biol Reprod. 1996;55:1055-62.

12. Johnson AL, Lee J. Granulosa cell responsiveness to follicle stimulating hormone during early growth of hen ovarian follicles. Poult Sci. 2016;95:108-14.

13. Tilly JL, Kowalski KI, Johnson AL. Stage of ovarian follicular development associated with the initiation of steroidogenic competence in avian granulosa cells. Biol Reprod. 1991;44:305-14.

14. Robinson FE, Etches RJ. Ovarian steroidogenesis during foillicular maturation in the domestic fowl (Gallus Domesticus). Biol Reprod. 1986;35:1096-105.

15. Johnson PA. Follicle selection in the avian ovary. Reprod Domest Anim. 2012;47:283-7.

16. Shao Y, Chen Y. Roles of circular RNAs in neurologic disease. Front Mol Neurosci. 2016;9:25.

17. Ashwal-Fluss R, Meyer M, Pamudurti NR, Ivanov A, Bartok O, Hanan M, Evantal N, Memczak S, Rajewsky N, Kadener S. circRNA biogenesis competes with pre-mRNA splicing. Mol Cell. 2014;56:5566.

18. Memczak S, Jens M, Elefsinioti A, Torti F, Krueger J, Rybak A, Maier L, Mackowiak SD, Gregersen LH, Munschauer M, Loewer A, Ziebold U, Landthaler M, Kocks C, le Noble F, Rajewsky N. Circular RNAs are a large class of animal RNAs with regulatory potency. Nature. 2013;495:333-8.

19. Hansen TB, Jensen TI, Clausen BH, Bramsen JB, Finsen B, Damgaard CK, Kjems J. Natural RNA circles function as efficient microRNA sponges. Nature. 2013;495:384-8.

20. Salzman J, Chen RE, Olsen MN, Wang PL, Brown PO. Cell-type specific features of circular RNA expression. PLoS Gene. 2013; 9.

21. Wang M, Yu F, Wu W, Zhang Y, Chang W, Ponnusamy M, Wang K, Li P. Circular RNAs: A novel type of non-coding RNA and their potential implications in antiviral immunity. Int J Biol Sci. 2017;13:1497506.

22. Wu Y, Xiao H, Pi J, Zhang H, Pan A, Pu Y, Liang Z, Shen J, Du J. The circular RNA aplacirc_13267 upregulates duck granulosa cell apoptosis by the apla-miR-1-13/THBS1 signaling pathway. J Cell Physiol. 2020;235:5750-63.

23. Shen M, Li T, Zhang G, Wu P, Chen F, Lou Q, Chen L, Yin X, Zhang T, Wang J. Dynamic expression and functional analysis of circRNA in granulosa cells during follicular development in chicken. BMC Genomics. 2019;20:96.

24. Glažar P, Papavasileiou P, Rajewsky N. circBase: a database for circular RNAs. RNA. 2014;20:166670.

25. Gao Y, Wang J, Zhao F. CIRI: an efficient and unbiased algorithm for de novo circular RNA identification. Genome Biol. 2015;16:4. 
26. Li L, Guo J, Chen Y, Chang C. X, C. Comprehensive CircRNA expression profile and selection of key CircRNAs during priming phase of rat liver regeneration. BMC Genomics. 2017;18:80.

27. Wang PL, Bao Y, Yee MC, Barrett SP, Hogan GJ, Olsen MN, Dinneny JR, Brown PO, Salzman J. Circular RNA is expressed across the eukaryotic tree of life. PloS one. 2014; 9.

28. Lu T, Cui L, Zhou Y, Zhu C, Fan D, Gong H, Zhao Q, Zhou C, Zhao Y, Lu D, Luo J, Wang Y, Tian Q, Feng Q, Huang T, Han B. Transcriptome-wide investigation of circular RNAs in rice. RNA. 2015;21:2076-87.

29. Salzman J. Circular. RNA expression: its potential regulation and function. Trends Genet. 2016;32:309-16.

30. Zhang Y, Zhang XO, Chen T, Xiang JF, Yin QF, Xing YH, Zhu S, Yang L, Chen LL. Circular intronic long noncoding RNAs. Mol Cell. 2013;51:792-806.

31. Kulcheski FR, Christoff AP, Margis R. Circular RNAs are miRNA sponges and can be used as a new class of biomarker. J Biotechnol. 2016;238:42-51.

32. Zhang X, Yan Y, Lei X, Li A, Zhang H, Dai Z, Li X, Chen W, Lin W, Chen F, Ma J, Xie Q. Circular RNA alterations are involved in resistance to avian leukosis virus subgroup-J-induced tumor formation in chickens. Oncotarget. 2017;8:34961.

33. Qiu L, Chang G, Bi Y, Liu X, Chen G. Circular. RNA and mRNA profiling reveal competing endogenous RNA networks during avian leukosis virus, subgroup J-induced tumorigenesis in chickens. PloS one. 2018; 13.

34. Wang Q, Leader A, Tsang BK. Follicular stage-dependent regulation of apoptosis and steroidogenesis by prohibitin in rat granulosa cells. J Ovarian Res. 2013;6:23.

35. Wojtysiak D, Okólski A, Sechman A. Structure and steroidogenic activity of the granulosa layer of F1 preovulatory ovarian follicles of the hen (Gallus domesticus). Folia Biol. 2011;59:59-64.

36. Johnson AL, Woods DC. Dynamics of avian ovarian follicle development: cellular mechanisms of granulosa cell differentiation. Gen Comp Endocrinol. 2009;163:12-7.

37. Dupont J, Reverchon M, Cloix L, Froment P, Rame C. Involvement of adipokines, AMPK, PI3K and the PPAR signaling pathways in ovarian follicle development and cancer. Int J Dev Biol. 2012;56:95967.

38. Zhang Z, Chen CZ, Xu MQ, Zhang LQ, Liu JB, Gao Y, Jiang H, Yuan B, Zhang JB. MiR-31 and miR-143 affect steroid hormone synthesis and inhibit cell apoptosis in bovine granulosa cells through FSHR. Theriogenology. 2019;123:45-53.

39. Du X, Zhang L, Li X, Pan Z, Liu H, Li Q. TGF- $\beta$ signaling controls FSHR signaling-reduced ovarian granulosa cell apoptosis through the SMAD4/miR-143 axis. Cell Death Dis. 2016;7:2476.

40. You S, Bridgham JT, Foster DN, Johnson AL. Characterization of the chicken follicle-stimulating hormone receptor (cFSH-R) complementary deoxyribonucleic acid, and expression of cFSH-R messenger ribonucleic acid in the ovary. Biol Reprod. 1996;55:1055-62.

41. Pfeffer PL, Sisco B, Donnison M, Somers J, Smith C. Isolation of genes associated with developmental competency of bovine oocytes. Theriogenology. 2007;68:84-90. 
42. Sirotkin AV, Benco A, Tandlmajerova A, Vasicek D, Kotwica J, Darlak K, Valenzuela F. Transcription factor p53 can regulate proliferation, apoptosis and secretory activity of luteinizing porcine ovarian granulosa cell cultured with and without ghrelin and FSH. Reproduction. 2008;136:611.

43. Panda S, Sato TK, Castrucci AM, Rollag MD, DeGrip WJ, Hogenesch JB, Provencio I, Kay SA. Melanopsin (Opn4) requirement for normal light-induced circadian phase shifting. Science. 2002;298:2213-6.

44. Wang Y, Lan Y, Lu H. Opsin3 Downregulation Induces Apoptosis of Human Epidermal Melanocytes via Mitochondrial Pathway. Photochem Photobiol. 2020;96:83.

45. Xu SR, Wei P, Yang QL, Jia GX, Ma SK, Yang QE, Jun Z, Zhang RN. Transcriptome analysis revealed key signaling networks regulating ovarian activities in the domestic yak. Theriogenology. 2020;147:50-6.

46. Naturil-Alfonso C, Peñaranda DS, Vicente JS, Marco-Jiménez F. Feed restriction regime in a rabbit line selected for growth rate alters oocyte maturation manifested by alteration in MSY2 gene expression. Reprod Domest Anim. 2017;52:976-84.

47. Gilbert AB, Evans AJ, Perry MM, Davidson MH. A method for separating the granulosa cells, the basal lamina and the theca of the preovulatory ovarian follicle of the domestic fowl (Gallus domesticus). Reproduction. 1977;50:179-81.

48. Langmead B, Salzberg SL. Fast gapped-read alignment with bowtie2. Nat. Methods. 2012;9:357-69.

49. Hansen TB, Venø MT, Damgaard CK, Kjems J. Comparison of circular RNA prediction tools. Nucleic Acids Res. 2016;44:58.

50. Gao Y, Zhang J, Zhao F. Circular RNA identification based on multiple seed matching. Brief Bioinform. 2017;19:803-10.

51. Yu G, Wang LG, Han Y, He QY. clusterProfiler: an R package for comparing biological themes among gene clusters. OMICS. 2012;16:284-7.

52. Wang X. miRDB: a microRNA target prediction and functional annotation database with a wiki interface. RNA. 2008;14:1012-7.

53. Wang K, Long B, Liu F, Wang JX, Liu CY, Zhao B, Zhou LY, Sun T, Wang M, Yu T, Gong Y, Liu J, Dong YH, Li N, Li PF. A circular RNA protects the heart from pathological hypertrophy and heart failure by targeting miR-223. Eur Heart J. 2016;37:2602-11.

54. Livak KJ, Schmittgen TD. Analysis of relative gene expression data using real-time quantitative PCR and the $2^{-\triangle \Delta C T}$ method. Methods. 2001;25:402-8.

\section{Figures}



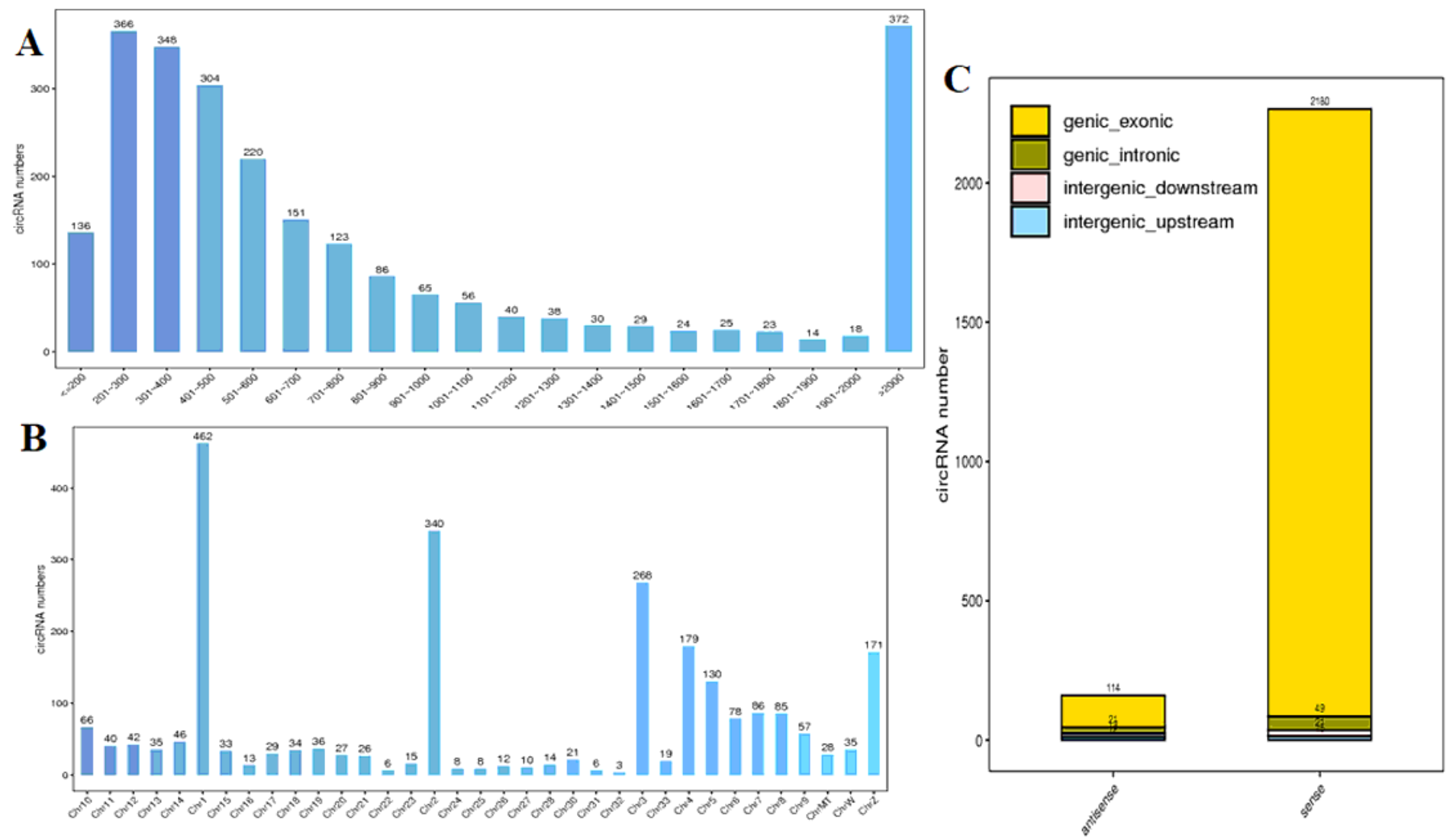

Figure 1

Profiling of circRNAs in GCs of SYFs of chickens under monochromatic light. (A) Splice length of circRNAs. (B) Chromosomes of circRNAs. (C) Source of circRNAs.
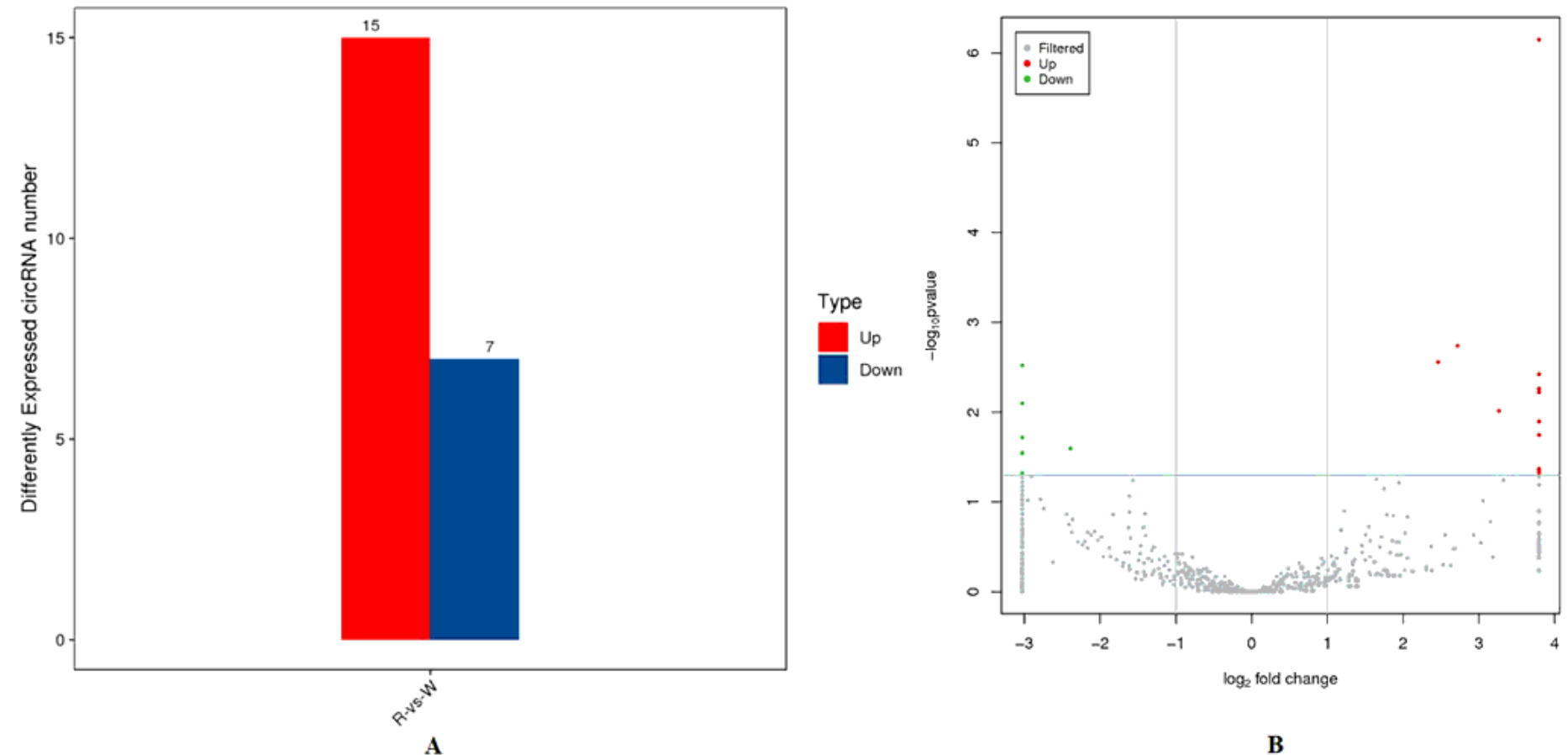

Figure 2 
Features of DE circRNAs. (A) Number of DE circRNAs. (B) Volcano plot of DE circRNAs.
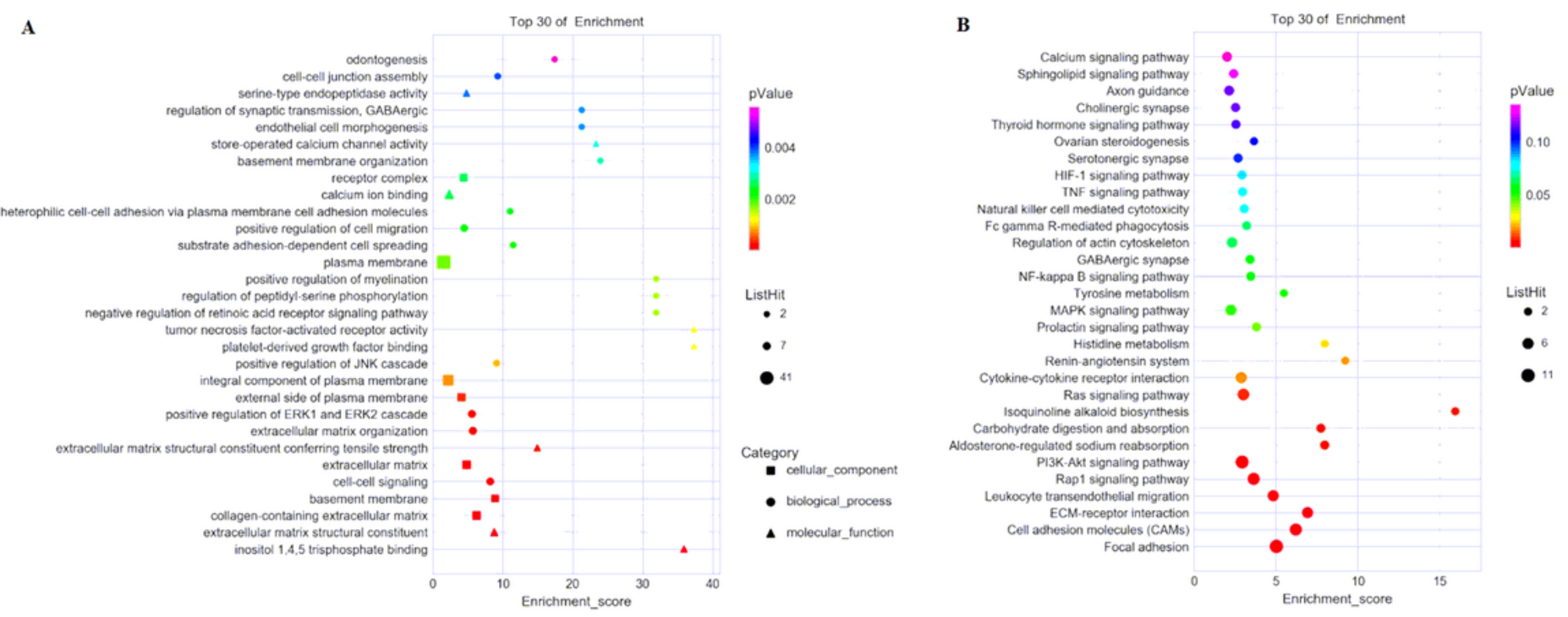

Figure 3

GO and KEGG analyses of host genes of DE circRNAs. (A) Top $10 \mathrm{GO}$ enrichment terms in BP, CC, and MF categories for host genes related to DE circRNAs. (B) Top 20 KEGG enrichment terms of host genes related to DE circRNAs.
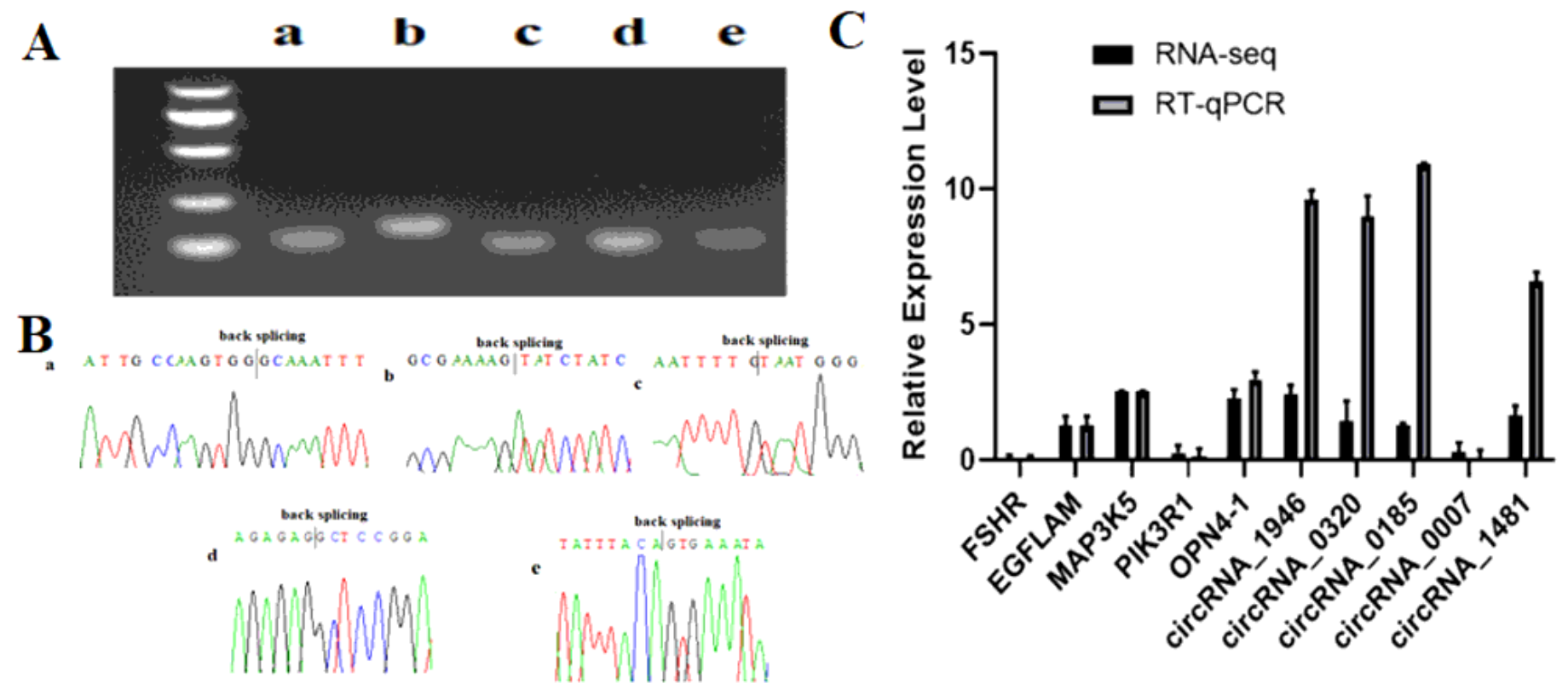

Figure 4

qRT-PCR analysis of circRNA expression. (A) Agarose gel electrophoresis of primers used to amplify circRNAs. (B) Sanger sequencing confirmation of the backsplicing junction of circRNAs. (C) RT-qPCR validation of differeDEcircRNAs in GCs of SYFs. a, b, c, d, and e represent circRNAs circRNA_1946, circRNA_0320, circRNA_0185, circRNA_0007, and circRNA_1481, respectively. 


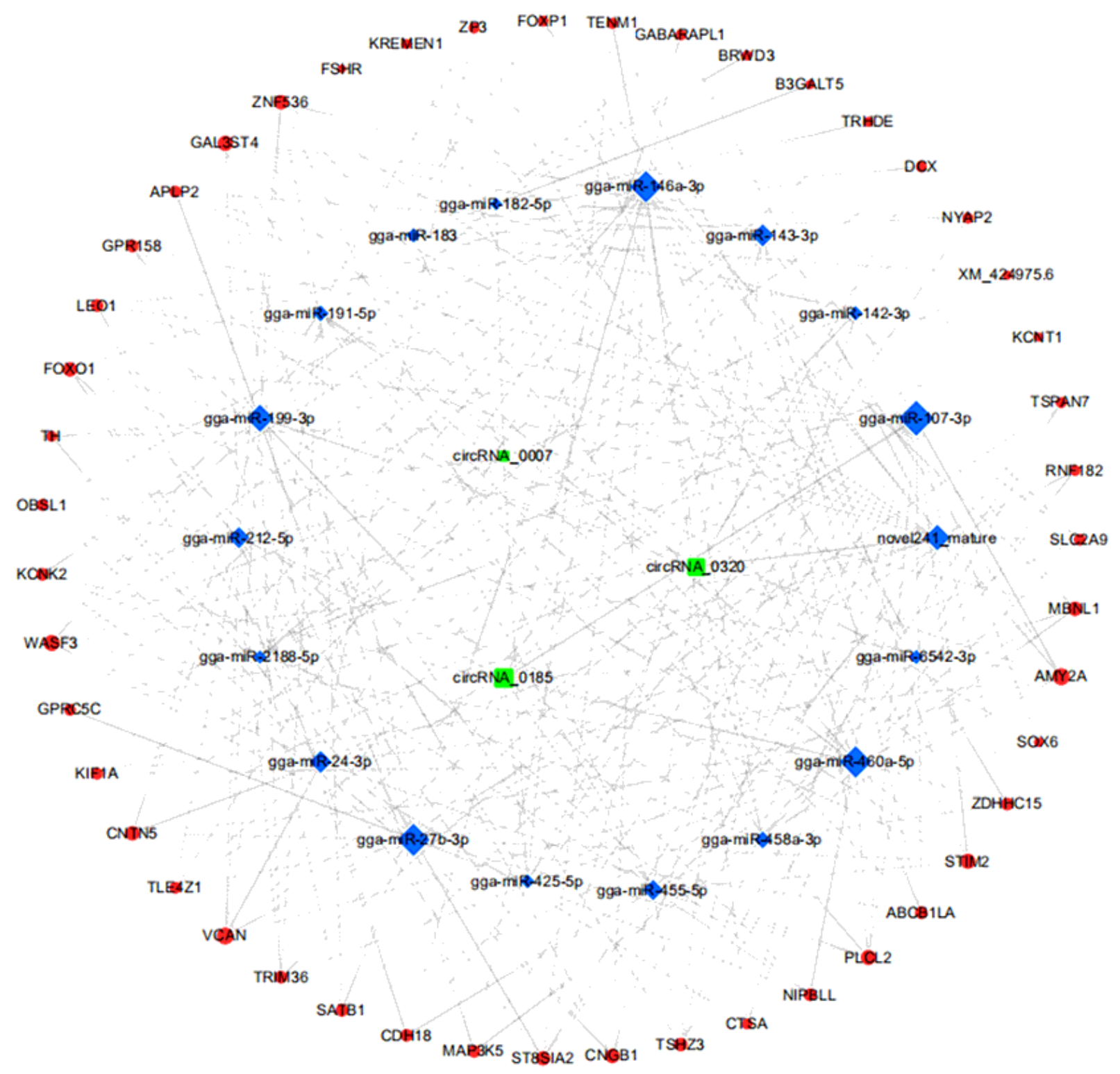

\section{Figure 5}

Network analysis of circRNA-miRNA-mRNA interactions.

\section{Supplementary Files}

This is a list of supplementary files associated with this preprint. Click to download.

- Figureofagarosegelelectrophoresis.jpg

- SupplementaryTableS6.xls

- SupplementaryTableS5.xls

- SupplementaryTableS4.xls

- SupplementaryTableS3.xls 
- SupplementaryTableS2.xls

- SupplementaryTableS1.xlsx 